\title{
Investigação da Estabilidade em Escoamento Bidimensional para o Fluido Oldroyd-B
}

\author{
Analice Costacurta Brandi ${ }^{1}$ \\ Ellen Silva Gervazoni ${ }^{2}$ \\ Faculdade de Ciências e Tecnologia, UNESP, Presidente Prudente, SP \\ Márcio Teixeira de Mendonça ${ }^{3}$ \\ Divisão de Propulsão Aeronáutica, IAE, São José dos Campos, SP \\ Leandro Franco de Souza ${ }^{4}$ \\ Instituto de Ciências Matemáticas e de Computação, USP, São Carlos, SP
}

\begin{abstract}
Resumo. Diversos escoamentos de interesse prático são de fluidos não-Newtonianos e uma classe destes fluidos é classificada como fluidos viscoelásticos. Muitas vezes é desejável saber se estes escoamentos propagam-se no estado laminar ou no turbulento. Para isto, utilizase a teoria de estabilidade linear (LST) para verificar a estabilidade dos escoamentos a perturbações não estacionárias de fluidos viscoelásticos. O modelo matemático adotado para o fluido não-Newtoniano do tensor extra-tensão é o Oldroyd-B. Neste trabalho, a teoria de estabilidade linear foi utilizada para investigar a convecção das ondas de Tollmien-Schlichting no escoamento de Poiseuille para o modelo de fluido Oldroyd-B. A fim de avaliar as curvas neutras de estabilidade, diferentes valores de parâmetros adimensionais são testados para os escoamentos de fluidos Newtoniano e não-Newtoniano. Os resultados numéricos obtidos são comparados com resultados da literatura.
\end{abstract}

Palavras-chave. Mecânica dos Fluidos, Teoria de Estabilidade Linear, Fluido Oldroyd-B, Equação de Orr-Sommerfeld.

\section{Introdução}

Há muitas aplicações científicas e industriais em que a estabilidade do escoamento laminar e a transição para a turbulência são relevantes. Sendo assim, é importante investigar a física da estabilidade e a transição laminar-turbulenta a fim de controlá-la, adiantá-la ou previni-la. Entre os diversos problemas surgidos nessa área está o tratamento de escoamentos de fluidos não-Newtonianos. Esse tipo de problema tem se tornado um grande desafio nessa área pois a maioria das aplicações industriais envolvem escoamentos de fluidos viscoelásticos, tais como, injeção de plásticos, processos de extrusão de forma geral e extração de petróleo.

\footnotetext{
${ }^{1}$ analice@fct.unesp.br

2 ellengerva@gmail.com

${ }^{3}$ marciomtm@iae.cta.br

${ }^{4}$ lefraso@icmc.usp.br
} 
Desse modo, existe um grande interesse em desenvolver métodos numéricos capazes de simular escoamentos de fluidos viscoelásticos para poder prever, a custos baixos e com bons resultados, o comportamento do escoamento de um fluido durante o processo industrial. A equação de Orr-Sommerfeld é uma equação diferencial fundamental para as perturbações (equação da estabilidade) que forma o ponto de partida para a teoria da estabilidade de escoamentos laminares [1].

A teoria de estabilidade hidrodinâmica investiga como essas perturbações são amplificadas ou amortecidas e como a evolução dessas perturbações estão relacionadas ao fenômeno de transição para o escoamento turbulento [4]. O desequilíbrio entre as forças de inércia e o campo de pressões pode levar a instabilidade se não houver efeitos viscosos dissipativos. Porém, a viscosidade também tem um efeito desestabilizador devido à difusão de quantidade de movimento.

Nesse contexto, este trabalho apresenta a análise de estabilidade linear, através da convecção de ondas de Tollmien-Schlichting, do escoamento de Poiseuille para o fluido Oldroyd-B. Para isso, será utilizada a teoria de estabilidade linear para analisar a estabilidade de escoamentos de fluidos viscoelásticos e comparar com a de fluidos Newtonianos, a fim de contribuir para uma melhor escolha dos parâmetros destes escoamentos.

\section{Formulação Matemática}

Considerando um escoamento incompressível, isotérmico e não-Newtoniano, as equações governantes são dadas pelas equações de continuidade e de Navier-Stokes, respectivamente,

$$
\begin{aligned}
\nabla \cdot \mathbf{u} & =0 \\
\rho\left(\frac{\partial \mathbf{u}}{\partial t}+\nabla \cdot(\mathbf{u u})\right) & =-\nabla p+\nabla \cdot \mathbf{T},
\end{aligned}
$$

em que u representa o campo de velocidade, $t$ é o tempo, $p$ é a pressão, $\rho$ é a densidade do fluido e $\mathbf{T}$ é o tensor extra-tensão. Neste trabalho, estudou-se escoamento viscoelástico em que a equação constitutiva utilizada é a do modelo Oldroyd-B dada por

$$
\mathbf{T}+\lambda \stackrel{\nabla}{\mathbf{T}}=2 \eta_{p} \mathbf{D}
$$

em que $\mathbf{D}=\frac{1}{2}\left(\nabla \mathbf{u}+(\nabla \mathbf{u})^{T}\right)$ é o tensor taxa de deformação, $\eta_{p}$ é um coeficiente de viscosidade polimérica, $\lambda$ refere-se ao tempo de relaxação do fluido e $\stackrel{\nabla}{\mathbf{T}}$ é a derivada convectada definida como

$$
\stackrel{\nabla}{\mathbf{T}}=\frac{\partial \mathbf{T}}{\partial t}+\nabla \cdot(\mathbf{u} \mathbf{T})-\mathbf{T} \cdot(\nabla \mathbf{u})^{T}-(\nabla \mathbf{u}) \cdot \mathbf{T} .
$$

Considerando um escoamento bidimensional, o conjunto de equações (1)-(3) foi adimensionalizado utilizando os seguintes parâmetros:

$$
\mathbf{x}^{*}=\frac{x}{L}, \quad \mathbf{u}^{*}=\frac{\mathbf{u}}{U}, \quad t^{*}=\frac{t U}{L}, \quad \mathbf{T}^{*}=\frac{\mathbf{T}}{\rho_{0} U^{2}}, \quad p^{*}=\frac{p}{\rho U^{2}},
$$


onde $L$ e $U$ denotam escala de comprimento e velocidade, respectivamente. Essas equações podem então ser escritas (omitindo o símbolo * por conveniência) como

$$
\begin{gathered}
\frac{\partial u}{\partial x}+\frac{\partial v}{\partial y}=0, \\
\frac{\partial u}{\partial t}+\frac{\partial u u}{\partial x}+\frac{\partial v u}{\partial y}=-\frac{\partial p}{\partial x}+\frac{\beta}{R e}\left[\frac{\partial^{2} u}{\partial x^{2}}+\frac{\partial^{2} u}{\partial y^{2}}\right]+\frac{\partial T^{x x}}{\partial x}+\frac{\partial T^{x y}}{\partial y}, \\
\frac{\partial v}{\partial t}+\frac{\partial u v}{\partial x}+\frac{\partial v v}{\partial y}=-\frac{\partial p}{\partial y}+\frac{\beta}{R e}\left[\frac{\partial^{2} v}{\partial x}+\frac{\partial^{2} v}{\partial y^{2}}\right]+\frac{\partial T^{x y}}{\partial x}+\frac{\partial T^{y y}}{\partial y}, \\
T^{x x}+W i\left(\frac{\partial T^{x x}}{\partial t}+\frac{\partial\left(u T^{x x}\right)}{\partial x}+\frac{\partial\left(v T^{x x}\right)}{\partial y}-2 T^{x x} \frac{\partial u}{\partial x}-2 T^{x y} \frac{\partial u}{\partial y}\right)=2 \frac{(1-\beta)}{R e} \frac{\partial u}{\partial x}, \\
T^{x y}+W i\left(\frac{\partial T^{x y}}{\partial t}+\frac{\partial\left(u T^{x y}\right)}{\partial x}+\frac{\partial\left(v T^{x y}\right)}{\partial y}-T^{x x} \frac{\partial v}{\partial x}-T^{y y} \frac{\partial u}{\partial y}\right)=\frac{(1-\beta)}{R e}\left(\frac{\partial v}{\partial x}+\frac{\partial u}{\partial y}\right), \\
T^{y y}+W i\left(\frac{\partial T^{y y}}{\partial t}+\frac{\partial\left(u T^{y y}\right)}{\partial x}+\frac{\partial\left(v T^{y y}\right)}{\partial y}-2 T^{x y} \frac{\partial v}{\partial x}-2 T^{y y} \frac{\partial v}{\partial y}\right)=2 \frac{(1-\beta)}{R e} \frac{\partial v}{\partial y},
\end{gathered}
$$

em que os parâmetros adimensionais $R e=\frac{\rho U L}{\eta_{0}}$ e $W i=\frac{\lambda U}{L}$ são associados aos números de Reynolds e Weissenberg, respectivamente. A quantidade de solvente Newtoniano é controlada através do coeficiente de viscosidade do solvente adimensional $\beta=\frac{\eta_{s}}{\eta_{0}}$, onde $\eta_{0}=\eta_{s}+\eta_{p}$ indica a viscosidade total do cisalhamento, e $\eta_{s}$ e $\eta_{p}$ representam as viscosidades do solvente Newtoniano e polimérico, respectivamente.

No problema do escoamento de Poiseuille viscoelástico o fluido está confinado dentro de um canal com paredes planas em $y=-1$ e $y=1$. Para o modelo Oldroyd-B, a velocidade do escoamento base coincide com a solução parabólica de Poiseuille, ou seja, $U=1-y^{2}$ e assume que todas as variáveis dependem apenas de $y$, a coordenada transversal do canal, exceto para a pressão cujo gradiente é constante na direção $x$. O componente da velocidade transversal do canal $v$ é identicamente nulo. As condições de contorno são adotadas no-slip nas paredes do canal.

\section{Teoria de Estabilidade Linear}

A teoria de estabilidade linear analisa o comportamento de um dado escoamento a perturbações de amplitude infinitesimal, como é apresentada para o escoamento de Poiseuille para o fluido Oldroyd-B. Esta teoria é baseada nas equações da continuidade e de Navier-Stokes, adotando algumas hipóteses sobre o escoamento e a forma de propagação das perturbações.

No caso bidimensional, o escoamento principal é tomado como permanente, tendo $u$ e $v$ como componentes cartesianos da velocidade, $p$ como a pressão e $T$ o tensor nãoNewtoniano. Os componentes da perturbação são muito pequenos em relação ao escoamento principal e, em um regime transiente, são representados por $\tilde{u}, \tilde{v}, \tilde{p}$ e $\tilde{T}$. Para análise no presente estudo toma-se um escoamento base invariante na direção $x$ e que o 
escoamento instantâneo pode ser decomposto em uma parte média e uma pequena perturbação. Portanto as variáveis podem ser compostas da seguinte forma, onde ( ${ }^{\sim}$ ) indica o escoamento perturbado

$$
\begin{array}{r}
u(x, y, t)=U(y)+\tilde{u}(x, y, t), \quad v(x, y, t)=\tilde{v}(x, y, t), \\
p(x, y, t)=P(y)+\tilde{p}(x, y, t), \quad T(x, y, t)=\mathbf{T}(y)+\tilde{T}(x, y, t) .
\end{array}
$$

As composições das variáveis dadas na equação (11) são substituídas nas equações (5)-(10). Então, subtraindo as equações que descrevem o escoamento base, obtém-se as equações para as perturbações. As condições de contorno adotadas para o problema do escoamento de Poiseuille são no-slip nas paredes e decaimento das perturbações longe das paredes. Uma vez que as equações resultantes são lineares e que os coeficientes das equações não dependem de $t$ e $x$, as soluções podem ser buscadas através do método de separação de variáveis, da seguinte forma:

$$
\begin{array}{ll}
\tilde{u}(x, y, t)=\bar{u}(y) e^{i(\alpha x-\omega t)}, & \tilde{v}(x, y, t)=\bar{v}(y) e^{i(\alpha x-\omega t)} \\
\tilde{p}(x, y, t)=\bar{p}(y) e^{i(\alpha x-\omega t)}, & \tilde{T}(x, y, t)=\bar{T}(y) e^{i(\alpha x-\omega t)}
\end{array}
$$

em que (-) representa a amplitude das perturbações, $i=\sqrt{-1}, \alpha=\alpha_{r}+i \alpha_{i}$ é o número de onda na direção $x$ e a taxa de crescimento espacial, $\omega=\omega_{r}+i \omega_{i}$ é a frequência angular e a taxa de crescimento temporal, onde $\lambda=2 \pi / \alpha$ é o comprimento de onda.

Substituindo as combinações lineares (12) nas equações de conservação para perturbação e reescrevendo de forma simplificada obtém-se a equação de Orr-Sommerfeld modificada para o fluido Oldroyd-B [2]

$$
\begin{aligned}
& \alpha(U-c)\left(\bar{v}^{\prime \prime}-\alpha^{2} \bar{v}\right)-\alpha \bar{v} U^{\prime \prime}+i \alpha^{2}\left(\bar{T}^{\prime x x}-\bar{T}^{\prime y y}\right)+\alpha\left(\bar{T}^{\prime \prime x y}+\right. \\
& \left.+\quad \alpha^{2} \bar{T}^{x y}\right)=\frac{i \beta}{R e}\left[\bar{v}^{I V}-2 \alpha^{2} \bar{v}^{\prime \prime}+\alpha^{4} \bar{v}\right] .
\end{aligned}
$$

A solução da equação de Orr-Sommerfeld corresponde a um problema de autovalor, cuja solução existe para alguns valores dos parâmetros $\alpha, \omega$ e $R e$, e depende do perfil de velocidade do escoamento base em questão. As perturbações analisadas são não estacionárias e propagam-se como ondas de Tollmien-Schlichting [5].

Note que quando $\omega$ é um número real e $\alpha$ um número complexo, a amplitude da perturbação cresce na direção do escoamento médio $x$ e a formulação é denominada formulação espacial. As componentes $\omega_{r}, \alpha_{r}$ e $\alpha_{i}$, representam, respectivamente, a frequência, o número de onda e a taxa de amplificação espacial. Quando $\alpha$ é um número real e $\omega$ um número complexo, a amplitude cresce no tempo e a formulação é denominada formulação temporal. Neste caso, $\omega_{i}$ é a taxa de amplificação temporal.

Um processo computacional implementado por Mendonça [3], adotando um Shooting method, é utilizado para resolver a equação de Orr-Sommerfeld.

\section{Resultados Numéricos}

Nesta seção é apresentada a análise de estabilidade do escoamento bidimensional de Poiseuille para um fluido viscoelástico do tipo Oldroyd-B. Os resultados numéricos da 
teoria de estabilidade linear são apresentados para a análise de estabilidade temporal e espacial. Quando avalia-se as curvas neutras de estabilidade, foca-se somente nas perturbações bidimensionais e compara-se os resultados de fluido Newtoniano com fluido Oldroyd-B para diferentes valores de parâmetros adimensionais.

\section{Análise temporal}

Aqui são apresentados resultados de análise temporal, portanto considera-se o $\alpha$ real e o $\omega$ complexo. Para verificação do código de teoria de estabilidade linear foram analisados resultados para diversos valores de Weissenberg, mantendo-se fixa a constante que controla a contribuição do solvente newtoniano no fluido $-\beta=0.9$. Estes estudos também foram realizados por Zhang et al. [6]. A Figura 1 mostra os valores obtidos para o número de Reynolds crítico para diferentes valores de Weissenberg. Reynolds crítico se refere ao menor valor de Reynolds onde o escoamento ainda é estável a perturbações não estacionárias. Pode-se observar uma boa concordância entre os resultados. Observa-se ainda que aumentando o Weissenberg de 0 a 3 o valor de Reynolds crítico diminui. À partir de Weissenberg igual a 3, observa-se que o valor de Reynolds crítico aumenta, mostrando que o escoamento pode se tornar mais estável a perturbações não estacionárias do que o escoamento newtoniano para números de Weissenberg maiores do que 7.

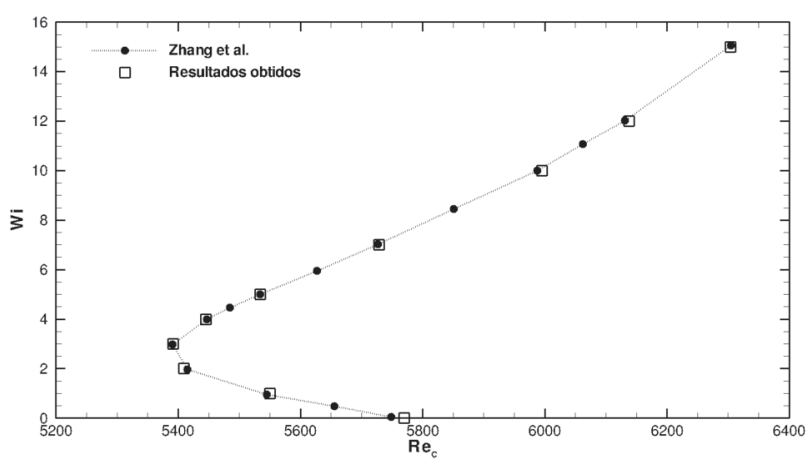

Figura 1: Valores de Reynolds crítico para diferentes valores de Weissenberg $-W i$. Constante fixa $\beta=0.9$.

E, também foram realizadas simulações para encontrar os valores de $\omega_{i}$ para diversos valores de Reynolds e $\alpha$. Para cada simulação é possível determinar a curva neutra, ou seja, onde os valores de $\omega_{i}=0$. Esta curva tem um formato característico de "banana", as taxas de amplificação menores do que zero $\omega_{i}<0$ estão do lado de fora da banana e as taxas de amplificação maiores do que zero $\omega_{i}>0$ estão no interior da mesma. A Figura 2 (a) apresenta curvas neutras para 3 diferentes valores da constante $\beta$, mantendo-se fixo o Weissenberg $W i=10.0$. Na mesma figura é apresentada a curva neutra para o fluido newtoniano. Pode-se observar que o comportamento do escoamento não é monotônico com a variação de $\beta$. Observa-se que o escoamento $\operatorname{com} \beta=0.9$ apresenta uma região estável maior do que a obtida para o escoamento newtoniano. Porém diminuindo-se mais o valor de $\beta$ a região instável tende a crescer. 
Para verificar a influência do número de Weissenberg na estabilidade do escoamento foram realizadas análises através da LST mantendo-se fixa a constante $\beta=0.9$. As curvas neutras foram obtidas e estão apresentadas na Figura 2(b). A mesma observação realizada na comparação feita na Figura 1 pode ser feita aqui. Pode-se observar que o valor de Reynolds crítico diminui com o aumento do Weissenberg até o valor igual a 3. À partir deste valor o Reynolds crítico diminui, até se tornar menor do que o encontrado para $W i=0$ para valores de $W i>7$.

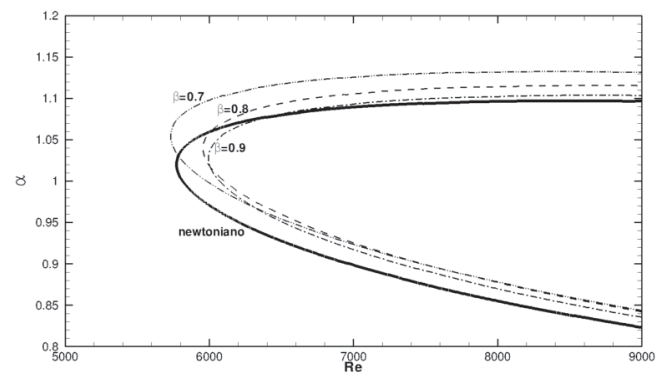

(a)

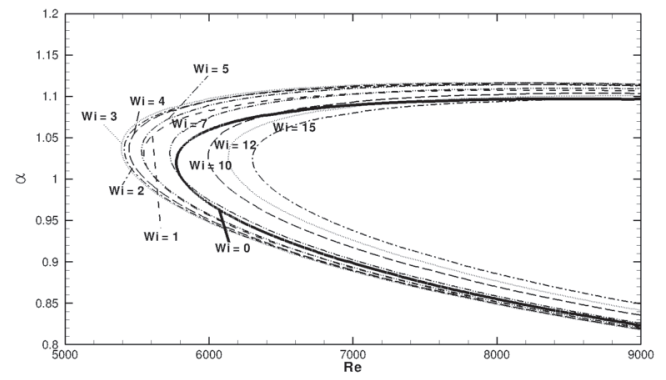

(b)

Figura 2: Análise temporal: comparação de curvas neutras para diferentes valores de $\beta$ e Weissenberg $W i$ : a) Número de Weissenberg fixo $-W i=10.0$ e b) Constante fixa $\beta=0.9$.

\section{Análise espacial}

Os resultados da análise espacial através da LST são apresentados nesta subseção. Neste caso considera-se o $\omega$ real e o $\alpha$ complexo. Realizou-se simulações para encontrar os valores de $\alpha_{i}$ para diversos valores de Reynolds e frequência $\omega_{t}$. Para cada simulação é possível determinar a curva neutra, ou seja, onde os valores de $\alpha_{i}=0$. Esta curva também apresenta o formato característico de "banana"e as taxas de amplificação maiores do que zero $\alpha_{i}>0$, ou seja, região estável, estão do lado de fora da banana e as taxas de amplificação menores do que zero $\alpha_{i}<0$ (região instável) estão no interior da mesma. A Figura 3(a) apresenta curvas neutras para 5 diferentes valores da constante $\beta$, mantendose fixo o Weissenberg $-W i=10.0$. A curva neutra para o fluido newtoniano também é apresentada na mesma figura. No caso da análise espacial pode-se observar também que o comportamento do escoamento não é monotônico com a variação de $\beta$. Observa-se que o escoamento com valores de $\beta=0.9$ e 0.8 apresenta uma região estável maior do que a obtida para o escoamento newtoniano. Porém diminuindo-se mais o valor de $\beta$ a região instável tende a crescer tornando-se bem mais instável do que o escoamento de fluido newtoniano.

$\mathrm{Na}$ análise espacial também foi verificada a influência do número de Weissenberg na estabilidade do escoamento através da LST. Nestes casos manteve-se fixa a constante $\beta=0.9$. A Figura 3(b) apresenta os resultados obtidos para as curvas neutras. Pode-se observar que o valor de Reynolds crítico diminui com o aumento do Weissenberg até o valor igual a 2.5. À partir deste valor o Reynolds crítico diminui, até se tornar igual ao obtido para $W i=0$ em $W i=7.5$. Para valores de Weissenberg maiores do que 7.5 observa-se que 
o escoamento apresenta uma região estável maior, com tendência a aumentar esta região quanto maior for o Weissenberg.

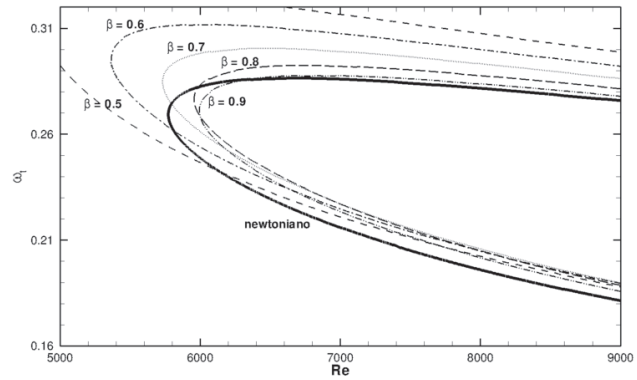

(a)

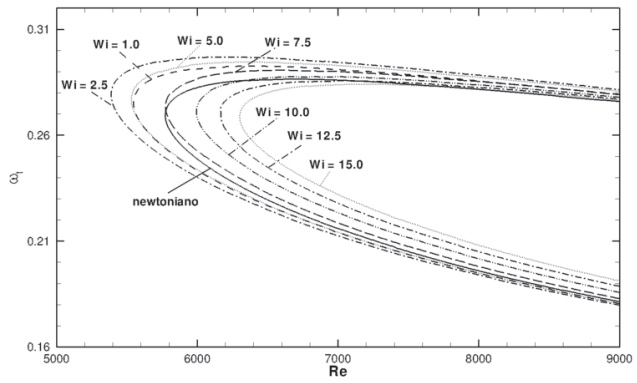

(b)

Figura 3: Análise espacial: comparação de curvas neutras para diferentes valores de $\beta$ e Weissenberg $W i$ : a) Número de Weissenberg fixo $-W i=10.0$ e b) Constante fixa $\beta=0.9$.

\section{Conclusões}

Neste trabalho a teoria de estabilidade linear foi utilizada para investigar a convecção das ondas de Tollmien-Schlichting no escoamento de Poiseuille para o modelo de fluido Oldroyd-B. A fim de avaliar as curvas neutras de estabilidade, diferentes valores de parâmetros adimensionais são testados para os escoamentos de fluidos Newtoniano e não-Newtoniano. Os resultados obtidos mostraram que os escoamentos tornaram-se mais instáveis a perturbações não-estacionárias à medida que $\beta$ diminuiu, e também que a taxa de crescimento dos escoamentos diminuiu com o número de Weissenberg.

\section{Referências}

[1] A. V. Boiko, A. V. Dovgal, G. R. Grek and V. V. Kozlov. Physics of Transitional Shear Flows, Springer, (2012).

[2] E. S. Gervazoni. Análise de estabilidade linear de escoamentos bidimensionais do fluido Oldroyd-B, Dissertação de Mestrado, Unesp, 2016.

[3] M. T. Mendonça e M. A. F. de Medeiros. Instabilidade hidrodinâmica e transição para turbulência com aplicações em engenharia e meteorologia. In Anais the Brazilian Congress of Thermal Sciences and Engineering (9th ENCIT), Caxambu, Minas Gerais, Brasil, 2002.

[4] A. Palmer. Linear Stability Analyses of Poiseuille Flows of Viscoelastic Liquids. Tese de Doutorado, The University of Wales, 2007.

[5] H. Schlichting. Boundary Layer Theory. McGraw-Hill, New York, (1968).

[6] M. Zhang, I. Lashgari, T. A. Zaki and L. Brandt. Linear stability analysis of channel flow of viscoelastic Oldroyd-B and FENE-P Fluids. J. Fluid Mech., 737:249-279, 2013. 